\title{
Conducting a Professional Telemedicine Visit Using High-Quality Webside Manner
}

\author{
Tania Elliott ${ }^{1}$ (1) Elizabeth C. Matsui ${ }^{2,3,4,5} \cdot$ Alison Cahill $^{6,5} \cdot$ Luke Smith $^{1} \cdot$ Lily Leibner $^{7}$ \\ Accepted: 23 December 2021 / Published online: 24 January 2022 \\ (c) The Author(s), under exclusive licence to Springer Science+Business Media, LLC, part of Springer Nature 2022
}

\begin{abstract}
Purpose of Review As telemedicine gains popularity among providers and patients alike, it is important to ensure the standards of care remain equivalent between the in-person and virtual settings. While bedside manner remains a key competency incorporated into medical school curricula, "webside manner," or professional standards for virtual care, remains less defined. Recent Findings Best practices exist including guidance prior to the visit, methods to maintain a professional background environment, and translation of core communication competencies for a video interaction. Through application of these practices, a provider can ensure the core interpersonal and communication competencies are fulfilled. These practices have direct application to allergy, asthma, and immunology care.

Summary This review provides an overview of best practices for professionalism and patient interaction for virtual care and examines specific applications to allergy, asthma, and immunology visits
\end{abstract}

Keywords Telehealth $\cdot$ Telemedicine $\cdot$ Virtual care $\cdot$ Webside manner $\cdot$ Video visit $\cdot$ Professionalism

\section{Introduction}

Telehealth has become an integral part of health services in the twenty-first century. A rise in technology across the globe meant that routine doctor's visits could become virtual, a new convenience for many patients. However, despite an already

This article is part of the Topical Collection on Telemedicine and Technology

Tania Elliott

Tania.Elliott@ascension.org

1 Virtual Care, Ascension, 4600 Edmundson Rd, MO 63134 St. Louis, USA

2 Department of Population Health, University of Texas at Austin Dell Medical School, Austin, TX, USA

3 Population Health and Pediatrics, University of Texas at Austin Dell Medical School, Austin, TX, USA

4 Center for Health and Environment: Education and Research, University of Texas at Austin Dell Medical School, Austin, TX, USA

5 Health Transformation Research Institute, University of Texas at Austin Dell Medical School, Austin, TX, USA

6 Department of Women's Health, University of Texas at Austin Dell Medical School, Austin, TX, USA

7 University of Virginia, Charlottesville, VA, USA increasing telehealth market, it was not until the COVID-19 pandemic swept the world that telehealth volumes soared $[1,2]$.

In the last week of March 2020, there was a 154\% increase in telehealth use, as compared to the same week from 2019 [3]. In 2020, it was predicted that virtual care would become a $\$ 250$ billion-dollar industry given that it provided both safe and effective care during the global pandemic. As of July 2021, telehealth use remains 38 times higher than use prior to the pandemic [4]. While both provider attitude and consumer satisfaction toward telemedicine has improved over the course of the pandemic, for telemedicine to remain successful and useful, providers and consumers must shift their perspectives and adapt to new ways of care.

Furthermore, health providers will continue to have to translate some of the core communication competencies and relationship building skills that occur in a brick and mortar setting into a virtual setting. This article provides best practices to assure the same standards of privacy, empathy, and cultural competency are met.

\section{Breaking Down Telehealth Barriers}

While adoption of virtual care has increased exponentially, with recent reports that it has stabilized at 38 times more utilization than pre-pandemic [4], barriers to long-term 
adoption still exist. There is still a perception on the part of both patients and clinicians that a physical examination cannot be completed virtually. However, general appearance, skin, extremity, musculoskeletal, neurologic, psychiatric, and portions of HEENT, respiratory, abdominal, and cardiovascular examination can be completed effectively [5]. The use of commercially available peripheral devices (stethoscope, otoscope, blood pressure cuff) allows for an even more complete evaluation. The examination, however, requires active patient participation, more so than is required in an office setting. It is important to instruct patients, professionally and respectfully, to participate in the patient-assisted exam. This requires comfort with technology on the part of the clinician, as well a modest ability to troubleshoot challenges, such as frame of the video image, adjustment of lighting, and angle of the camera. A show-and-tell approach, demonstrating what the patient will need to do, is helpful for patients and can support relationship and rapport building. A physical exam may or may not be required for an allergy visit. For example, a history of accidental ingestions and other allergy history are the key pieces of information required for a patient coming in for a food allergy follow-up and renewal of an epinephrine autoinjector. For a patient with chronic urticaria, a skin exam can be completed either by video or through photo uploads.

Another barrier to long-term implementation of virtual care relates to workflow. Much of a clinician's practice is set up to be delivered in a brick and mortar setting. Therefore, attention needs to be paid to the workflow changes that are needed in order to support visits when the patient and/or the clinician are not physically in the office. This also requires expectation setting for staff members, so that, when a virtual visit is offered, it is done so confidently to reassure patients that the quality of care will not be compromised. Clear expectations also assist staff in understanding which visits require in-person care (e.g., skin testing, PFTs) and which can be conducted virtually (anything that is not procedure based). It may be helpful to adopt the approach that virtual care is part of the standard of care - there is not a "wrong time" for a clinician and patient to look one another in the eye, share information about a health condition, provide advice, and make recommendations on next steps. While it is true that sometimes patients show up to the wrong site of care, such as the office when they need an emergency room or vice versa, there is still value in interacting with the patient, triaging them, and educating them on symptom monitoring and about their overall condition. Adopting a virtual-forward approach can help avoid confusion when staff is booking appointments for patients. Table 1 illustrates examples of visits best suited for telemedicine versus in-person care.
Reimbursement concerns can also impact the way clinicians and practices communicate about virtual visits. Setting expectations regarding cost sharing/copays and how these will be collected should be addressed with patients in advance of the visit. It is also important to keep up to date with telemedicine reimbursement policies for commercial payors, Medicare, and Medicaid. State licensure requirements are another barrier to virtual care adoption. During the public health emergencies, many states waived state licensure requirements. However, as a sense of normalcy returns, clinicians should consider applying for neighboring state licenses to assure that out-of-state patients can still benefit from virtual visits.

Lastly, there may be concerns regarding health equity. Clinicians should acknowledge that there may be assumptions, based in fact or not, that can influence which patients are offered virtual visits. While there was a perception that virtual care is an offering mainly for younger, tech-savvy patients, we now know that even geriatric patients are satisfied with virtual visits [6, 7]. Based on an August 2020 survey, $72 \%$ of older adults said that they would be interested in scheduling a telemedicine appointment with their provider. Out of the older adult population that did have a telemedicine visit during the pandemic, 91\% reported high satisfaction and ease in connecting with their doctor [7]. That said, attention needs to be paid to individuals not as comfortable using a smartphone or computer and those requiring special accommodations due to hearing or visual impairment. It can be helpful in these scenarios to also engage a family member or caregiver in advance of and during the visit. Ultimately, by empowering providers to offer video visits through defined workflows and accessible technology offerings, clinicians can recommend the best method of care delivery and remove the onus from the patient to choose which service is more appropriate.

It is important to give patients choice, and problem solve barriers alongside them, as opposed to assuming a patient would not want or be able to have a virtual visit. There are also populations that may not have access to broadband and tend to be poorer

Table 1 Care best suited for in-person versus virtual visits

\begin{tabular}{ll}
\hline In-person visit & Virtual visit \\
\hline - Allergy shots & • Immunodeficiency* \\
- Pulmonary function testing (PFT) & - Asthma \\
& - Urticaria \\
- Endoscopy & - Atopic dermatitis \\
- Skin or intradermal testing & - Allergic rhinitis \\
- Oral food challenges & • Food allergy* \\
- Drug challenges & • Allergen immuno- \\
& therapy 6mo/yearly \\
& check in \\
\hline
\end{tabular}

*Lends itself well to new patient intake 
and be more likely to be Black, Latino/a, and of other ethnic minority populations. Their limited access - relative to their less poor, non-ethnic minority counterparts - could perpetuate if not exacerbate racial and ethnic health disparities. These locations also tend to be rural with limited access to clinicians. For these populations, partnering with communities and schools to offer virtual visits may be a useful option. When it comes to access and digital literacy, partner with patients to solve access challenges. This can help to strengthen the clinician-patient relationship and avoid biases.

\section{Webside Manner Best Practices}

\section{Before the Visit}

\section{Appearance, Professional Dress, and Background Environment}

Providers should dress as they would when they see a patient in person, such as a white coat, business casual clothes, or scrubs. Appearance sets the tone for the visit and can help patients overcome skepticism around whether a virtual visit can be equally as good as in-person care.

Room setup and background are also important to ensuring a high-quality experience for patients. The background should be clean and ideally face a wall that has a photograph, diploma, or bookshelf. Avoid areas where people may be walking in the background, as this would convey to the patient that the area is not private. Some clinicians place a meaningful item in the background, such as a picture, sculpture, or book, that can sometimes spark conversation with the patient. If an ideal physical background is not available, many video conferencing platforms offer virtual backgrounds.

Computers should be set up in a well-lit environment, ideally in front of a window. Alternatively, a light can be purchased that attaches to the computer to assure consistent lighting. Providers should ensure their face is framed within the middle of the camera viewing area and at an appropriate distance prior to beginning the visit. Figure 1 shows an example of an ideal room setup.

Wearing headphones is also a way to show the patient that the conversation is confidential. Furthermore, the provider should set up their computer screen with all charts and information needed prior to the visit to minimize toggling between windows during the visit. Table 2 emphasizes best practices for conducting a virtual visit.

\section{Expectation Setting}

Clear communication and expectation setting with patients is key to integrate virtual visits as part of routine practice.

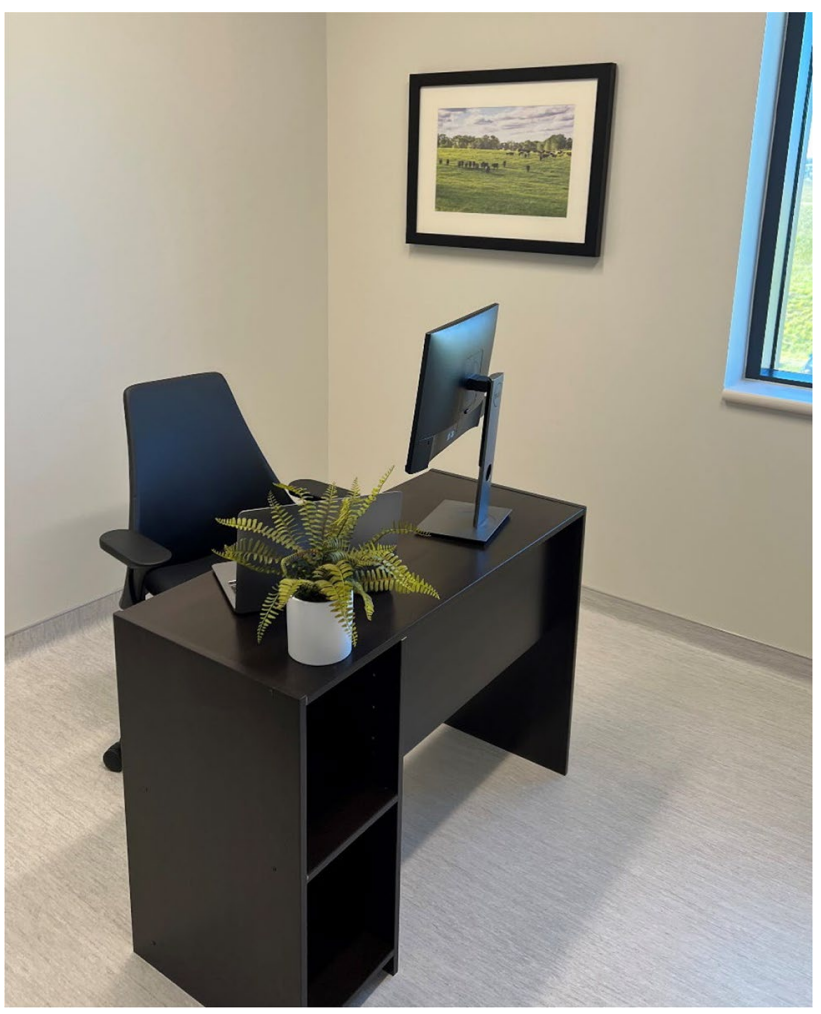

Fig. 1 Example of an ideal telemedicine setup

In-office flyers and handouts can be utilized to drive awareness of virtual visit offerings. A simple reference guide for patients on how to prepare for and connect to a visit can also be a helpful addition to a virtual practice. Table 3 illustrates an example checklist providers and staff may utilize.

When scheduling or confirming appointments, several key items should be addressed. Will medical translation services be required? Would the patient like a family member or caregiver to join the visit? Patients should be reminded or invited to test their technology prior to the visit, including their browser settings, camera, and microphone. In the event of technical issues, patients should know who to contact. Ideally there is an individual in the office who could easily troubleshoot some of the most common challenges. Finally, a patient should be clear on how (text message, email, etc.) and when they will receive a visit link to join their appointment. For the first virtual visit, it might be beneficial to do a test visit with the patient in conjunction with an appointment confirmation outreach.

On visit day, in the event the clinician is running behind schedule, the patient should be made aware. Patients often may think they are in the wrong place or have clicked the wrong link if a clinician has not joined right away. If the telemedicine technology does not have a virtual waiting room, one option would be to have a staff member connect with the patient via a video visit first, to re-create a check-in process 
Table 2 Tips for conducting a professional virtual visit

\begin{tabular}{|c|c|}
\hline Physic & Patient engagement: compassionate and personal \\
\hline $\begin{array}{l}\text { Room } \\
\text { - Ensure a quiet and well-lit room } \\
\text { - Avoid large rooms and rooms without carpeting to minimize echo } \\
\text { Backdrop } \\
\text { - Ensure a neutral and professional backdrop } \\
\text { - Avoid virtual backgrounds } \\
\text { - Do not have a window or other bright light behind you } \\
\text { Camera view } \\
\text { - Position camera approximately } 2 \text { feet from your face } \\
\text { - Ensure no PHI is visible } \\
\text { - Do not have an open door visible in the visit }\end{array}$ & $\begin{array}{l}\text { Appearance and body language } \\
\text { - Dress professionally as you would in your clinic } \\
\text { - Avoid eating during visits } \\
\text { - To engage in eye contact, look at your camera, not your screen } \\
\text { Communication } \\
\text { - Introduce yourself to patient (and medical interpreter if present). Ask the } \\
\text { patient how they would like to be addressed } \\
\text { - Verify you can see and hear each other and confirm a call back number if } \\
\text { there are technical issues } \\
\text { - Practice narration of care - if you are typing notes or looking up patient } \\
\text { history, verbalize what you are doing }\end{array}$ \\
\hline
\end{tabular}

(insurance verification, demographic information changes, changes to medical history and/or medications, etc.), and then conference in the clinician once the intake is complete. Non-clinical care team members are integral to the success of integrating virtual care into practice as they are on the front lines of care, and can impact a patient's impression on the value, appropriateness, or ease of conducting a virtual visit.

\section{During the Visit}

There has been a perception that a clinician-patient relationship cannot be built or maintained with virtual visits. With appropriate training and guidance, clinicians can develop strong relationships with their patients in a way that augments the relationship that exists within the four walls of an office.

\section{Building Rapport}

A random survey sample of the adult US population showed a preference for telemedicine care delivery by clinicians with whom they have an established relationship. Fifty percent of respondents were willing to see their own primary care provider via telemedicine, whereas only $17 \%$ reported willingness to see a provider from an unaffiliated health care organization [8]. This highlights the perceived importance of the clinician-patient relationship in virtual care. A similar study looking at patient perceptions of exceptional interactional skills via telemedicine found that patients often value a clinician that is caring and concerned, using non-verbal gestures to convey that sentiment [9]. This involves making eye contact with the patient by looking directly into the camera and having appropriate facial expressions. It is important to note that a camera and lighting can exaggerate facial expressions, so this should be considered. A useful tip is for the clinician to look at their own facial expressions through the video platform while speaking with the patient and adjust in real time.

Acknowledging limitations of the visit and setting the stage for what to expect is especially important for patients new to virtual care who may have hesitation. Patient comfort and privacy is of utmost importance. Unlike a medical office that is specifically designed to ensure privacy, a patient engaged in a virtual visit may worry about household members listening in on the call (on both the patient and provider end) or be uncomfortable sharing sensitive content over video or image with a provider who is not physically with them. Providers should explain to the patient that the visit is not being recorded, the video will not be shared or posted anywhere, and all aspects of the visit meet HIPAA compliance guidelines.

Providers should determine in advance whether they will actively document during the encounter or wait until afterwards. Such a decision may be made in consultation with the patient - some patients may find typing/clicking noises distracting and request it be saved until after the encounter. The same considerations should be taken when using multiple windows to access the patient's chart or verify recent guidelines. In many cases, acknowledging and
Table 3 Example "before the visit" checklist for providers and staff

\begin{tabular}{ll}
\hline Yes & Bofore the visit - patient considerations \\
\hline & Will medical translation services be required? \\
& Would the patient like a family member or caregiver to join the visit? \\
& Has the patient's technology been tested (browser settings, camera, and microphone)? \\
& Does the patient know who to contact in the event of technical difficulties? \\
& Is the patient aware how they will receive the visit link (text message, email, etc.)? \\
\hline
\end{tabular}


explaining behaviors that may be perceived as rude (typing as the patient speaks or not making eye contact due to screen setup, for example) can set a patient's mind at ease and ensure patients are aware the provider is placing their care at the forefront.

\section{Sharing Information/Providing Guidance}

Without having the familiar environment of a doctor's office, providers may sometimes need to over communicate the care plan for patients. Patients may require coaching throughout the visit to assist with the physical exam. For example, the provider may wish to explain the physical exam in piecemeal format allowing the patient to follow the directions step by step. In doing this, the provider can ensure a higher quality exam and place the patient - especially one who is experiencing virtual care for the first time - at ease.

Much like an in-person visit, helping a patient understand their condition and symptoms leads to better outcomes and improved quality of care. This includes stating a review of what was done, the findings of the examination, treatment plan, and next steps. Allocating a portion of the visit for the patient to ask questions can be a valuable way to ensure understanding of the agreed-upon treatment plan while also providing an opportunity for the patient to voice any lingering concerns. It can be off putting to patients to "hang up" or "end" the visit, so the shared decision-making, follow-up, treatment plans, and next steps need to be actively communicated, as well as anything the patient can expect to receive at the conclusion of the visit, such as a care plan through a patient portal or notice of a follow-up appointment, for example.

\section{Shared Decision-making}

Video visits allow providers the opportunity to peer into a patient's home or living situation in a way they could not experience in the face-to-face setting. In working with the patient to develop an ideal treatment plan, the provider can consider their situation and nuances within their home that may present challenges. Additionally, video visits allow for an easy connection to caregivers, friends, and family members who may want to participate in the conversation. Most video visit platforms allow for at least 4 participants in a call. This is particularly useful in older patients who may not have loved ones nearby. Additionally, video conferencing can be utilized to bring in other care team members, such as PCPs, case managers, pharmacists, therapists, or other specialists who may be involved in the patient's care. Table 4 summarizes best practices to develop exceptional webside manner.

\section{Applications for Allergy, Asthma, and Immunology Care}

Telehealth provides unique opportunities for delivery of allergyimmunology care. For example, a video tour of the pantry allows for individually tailored food avoidance education. Allergistsimmunologists can "co-read" ingredient labels with their patients, using the food items that they commonly eat. This approach also provides an opportunity to engage family members who might otherwise not be able to attend an in-person clinic visit in a discussion about interpreting food ingredient labels. The telehealth format also allows for a joint trip to the grocery store, which would provide another opportunity to co-read ingredient labels and discuss assessing risks of foods in bulk containers or in buffets, tailored to the patient's specific food allergies. For parents of children with food allergies, allergists-immunologists can more concretely discuss strategies for storing and tagging foods in their kitchen to minimize the risk of accidental ingestion. Allergists-immunologists can help parents identify areas of the pantry or kitchen that are not reachable by smaller children for storage of unsafe foods and develop
Table 4 Webside manner best practices

\begin{tabular}{ll}
\hline $\begin{array}{l}\text { Interpersonal and communication compe- } \\
\text { tencies }\end{array}$ & How to achieve through webside manner \\
\hline Building rapport & - Explain virtual visit limitations \\
& - Discuss importance of shared decision-making \\
& - Ensure comfort and privacy \\
& - Ensure direct eye contact \\
Active listening & - Verbalize what is being seen and done \\
& - Remain engaged and conversational \\
& - Acknowledge and explain actions \\
Sharing information/providing guidance & - Do not be afraid to over communicate \\
& - Allocate time for questions and discussion \\
& - Set expectations for end-of-visit and follow-up \\
& - Mutually develop a plan \\
Shared decision-making & - Provide options based on preferences \\
& - Take into account the patient's situation \\
& - Include caregivers and/or care team members on the visit
\end{tabular}


approaches to tagging foods - for example, with colored stickers - to indicate safe and unsafe foods that work best in the patient's own home. Such offerings could be billed used existing procedure codes. However, opportunities exist to advocate for broader counseling codes that relate more directly to offerings available through telehealth.

For patients with allergic rhinitis or asthma, a view into the home can reveal sources of allergen and pollutant exposure that are not easily obtained with an environmental history. For example, a direct view of a child's bed can reveal many stuffed animals or many bedding components (blankets, comforters, etc.) that would all need washing to reduce dust mite allergen exposure. A home telehealth visit also facilitates medication reconciliation. Patients with asthma often have multiple inhalers that they have saved as they have transitioned from one controller medication to another. Directly visualizing those medications with the patient and walking through how the patient is using each inhaler can be very informative. Because patients do not commonly bring their medications to an in-person clinic visit, a home telehealth visit provides an opportunity to get a more accurate medication history and to provide patient education with their medications on hand. A home medication reconciliation may also reveal that the spacer is lost or is cracked and needs to be replaced.

Patient preference should be considered as some may not welcome what is essentially a home visit. A thoughtful discussion with the patient prior to the telehealth home visit can give the patient an opportunity to consider the potential benefits and prepare for the visit. The above telehealth strategies for allergic disorders have not been well studied. For example, there have been no studies that have systematically assessed patient preference and satisfaction with telehealth home visits, whether for food allergy or asthma or allergies. Whether some of the above approaches are more effective at improving patient knowledge or allergic disease outcomes than in-person visits is unclear. However, there are decades of experience conducting in-person home visits for asthma, illustrating that many patients find them valuable. These home visits have been conducted mostly among children living in poverty, so this experience may not apply to other populations. Understanding the patient preferences along with the effectiveness of these approaches is particularly important given the recent explosion of telehealth. Moreover, even though the digital divide has been shrinking, it persists and clinicians risk perpetuating, if not exacerbating, disparities in allergic disorders if deliberate steps are not taken to measure and address inequities in telehealth access.

\section{Conclusions}

Spurred by the COVID-19 pandemic and rapidly improving technology, virtual care is now becoming part of the standard of care. Yet, it is important to acknowledge that this type of care delivery has a learning curve. It will be important to incorporate virtual care training into medical school and graduate medical education and to continue to collect data on patient and provider satisfaction and clinical outcomes.

\section{Compliance with Ethical Standards}

Conflict of Interest Tania Elliott, Elizabeth C. Matsui, Alison Cahill, Luke Smith, and Lily Leibner declare that they have no conflict of interest.

Human and Animal Rights and Informed Consent This article does not contain any studies with human or animal subjects performed by any of the authors.

\section{References}

1. Betancourt JA, Rosenberg MA, Zevallos A, Brown JR, Mileski M. The impact of COVID-19 on telemedicine utilization across multiple service lines in the United States. Healthcare. 2020;8:380. https://doi.org/10.3390/healthcare8040380.

2. Becker CD, Forman L, Gollapudi L, Nevins B, Scurlock C. Rapid implementation and adaptation of a telehospitalist service to coordinate and optimize care for COVID-19 patients. Telemedicine and e-Health. 2021;27:388-96. https://doi.org/10.1089/tmj.2020.0232.

3. Koonin LM, Hoots B, Tsang CA, Leroy Z, Farris K, Jolly B, et al. Trends in the use of telehealth during the emergence of the COVID-19 pandemic - United States, January-March 2020. MMWR Morb Mortal Wkly Rep. 2020;69:1595-9. https://doi.org/ 10.15585/mmwr.mm6943a3.

4. Bestsennyy O, Gilbert G, Harris A, Rost J. Telehealth: a quartertrillion-dollar post-COVID-19 reality? In: McKinsey \& Company. 2021. https://www.mckinsey.com/industries/healthcare-systemsand-services/our-insights/telehealth-a-quarter-trillion-dollar-postcovid-19-reality. Accessed 31 Aug 2021.

5. Benziger CP, Huffman MD, Sweis RN, Stone NJ. The telehealth ten: a guide for a patient-assisted virtual physical examination. Am J Med. 2021;134:48-51. https://doi.org/10.1016/j.amjmed.2020.06.015.

6. Murphy RP, Dennehy KA, Costello MM, Murphy EP, Judge CS, O'Donnell MJ, et al. Virtual geriatric clinics and the COVID-19 catalyst: a rapid review. Age Ageing. 2020;49:907-14. https://doi. org/10.1093/ageing/afaa191.

7. Gavin K. Telehealth visits have skyrocketed for older adults but concerns, barriers remain. In: University of Michigan News. 2020. https://news.umich.edu/telehealth-visits-have-skyrocketed-forolder-adults-but-concerns-barriers-remain/. Accessed 31 Aug 2021.

8. Welch BM, Harvey J, O'Connell NS, McElligott JT. Patient preferences for direct-to-consumer telemedicine services: a nationwide survey. BMC Health Serv Res. 2017. https://doi.org/10. 1186/s12913-017-2744-8.

9. Elliott T, Tong I, Sheridan A, Lown BA. Beyond convenience: patients' perceptions of physician interactional skills and compassion via telemedicine. Mayo Clin Proc Innov Qual Outcomes. 2020;4:305-14. https://doi.org/10.1016/j.mayocpiqo.2020.04.009.

Publisher's Note Springer Nature remains neutral with regard to jurisdictional claims in published maps and institutional affiliations. 